\title{
Observations on the Cerebral Effects of Refractory Intracranial Hypertension After Severe Traumatic Brain Injury
}

Joseph Donnelly ${ }^{1,2^{*}} \mathbb{D}$, Peter Smielewski ${ }^{1}$, Hadie Adams ${ }^{3}$, Frederick A. Zeiler ${ }^{4,5,6,7} \mathbb{D}$, Danilo Cardim ${ }^{8}$, Xiuyun Liu', Marta Fedriga', Peter Hutchinson ${ }^{3,9}$, David K. Menon ${ }^{4,9}$ and Marek Czosnyka 1,10

(c) 2019 The Author(s)

\begin{abstract}
Background: Raised intracranial pressure (ICP) is a prominent cause of morbidity and mortality after severe traumatic brain injury (TBI). However, in the clinical setting, little is known about the cerebral physiological response to severe and prolonged increases in ICP.

Methods: Thirty-three severe TBI patients from a single center who developed severe refractory intracranial hypertension (ICP > $40 \mathrm{~mm}$ Hg for longer than $1 \mathrm{~h}$ ) with ICP, arterial blood pressure, and brain tissue oxygenation $\left(\mathrm{P}_{\mathrm{BT}} \mathrm{O}_{2}\right)$ monitoring (subcohort, $n=9$ ) were selected for retrospective review. Secondary parameters reflecting autoregulation (including pressure reactivity index-PRx, which was available in 24 cases), cerebrospinal compensatory reserve (RAP), and ICP pulse amplitude were calculated.

Results: PRx deteriorated from $0.06 \pm 0.26$ a.u. at baseline levels of ICP to $0.57 \pm 0.24$ a.u. $(p<0.0001)$ at high levels of ICP (> $50 \mathrm{~mm} \mathrm{Hg}$ ). In 4 cases, PRx was impaired (>0.25 a.u.) before ICP was raised above $25 \mathrm{~mm} \mathrm{Hg}$. Concurrently, $\mathrm{P}_{\mathrm{BT}} \mathrm{O}_{2}$ decreased from $27.3 \pm 7.32 \mathrm{~mm} \mathrm{Hg}$ at baseline ICP to $12.68 \pm 7.09 \mathrm{~mm} \mathrm{Hg}$ at high levels of ICP $(p<0.001)$. The pulse amplitude of the ICP waveform increased with increasing ICP but showed an 'upper breakpoint' - whereby further increases in ICP lead to decreases in pulse amplitude - in 6 out of the 33 patients.
\end{abstract}

Discussion: Severe intracranial hypertension after TBI leads to decreased brain oxygenation, impaired pressure reactivity, and changes in the pulse amplitude of ICP. Impaired pressure reactivity may denote increased risk of developing refractory intracranial hypertension in some patients.

Keywords: Traumatic brain injury, Intracranial pressure, Cerebral hemodynamics, Autoregulation, Cerebral oxygenation, Cerebral perfusion pressure, Intracranial hypertension

\section{Introduction}

Raised intracranial pressure (ICP) can occur due to an expanding mass lesion or due to increases in volume of any of the vascular, cerebrosipinal fluid (CSF) or parenchymal compartments within brain [1]. The detrimental effects of raised ICP are twofold. One is the development of transtentorial pressure gradients that can cause focal ischemia of vital brain stem centers leading to rapid death. The second is that increased ICP causes an increase in cerebral venous pressure by compression at the level of the bridging veins, leading in turn to a

*Correspondence: joseph.donnelly@cantab.net

2 Department of Anaesthesiology, Level 12 Auckland Support Building,

Auckland City Hospital, University of Auckland, 2 Park Road, Grafton,

Auckland, New Zealand

Full list of author information is available at the end of the article

\section{Springer}


decrease in cerebral perfusion pressure (CPP) and, if CPP decreases below the lower limit of autoregulation, global cerebral hypoperfusion $[2,3]$.

Surprisingly, the cerebral physiological sequelae of raised ICP secondary to traumatic brain injury (TBI) are unclear. As previously shown, increased ICP decreases CPP, which with dysfunctional autoregulation, causes a decrease in cerebral blood flow, raises arterial blood pressure (ABP), and modifies the ICP pulse waveform [4]. However, whether these findings translate to the complex situation of raised ICP after severe TBI is uncertain.

In this study, we sought to describe the cerebral oxygenation, cerebrovascular pressure reactivity, and ICP pulse amplitude response to severe and sustained intracranial hypertension after severe TBI.

\section{Methods}

\section{Study Design and Setting}

This study was conducted as a retrospective analysis of a prospectively maintained database cohort (1992-2017), in which high-frequency physiological monitoring data had been archived. Monitoring of brain modalities was conducted as a part of standard patient care and archived in an anonymized database of physiological monitoring. Data on age, injury severity, and clinical status were recorded at the time of monitoring on this database, and no attempt was made to re-access clinical records for additional information. Since all data were extracted from the hospital records and fully anonymized, no data on patient identifiers were available, and therefore, formal patient or proxy consent and institutional ethics approval were not required.

\section{Participants}

TBI patients with a clinical need for ICP monitoring and computerized signal recordings were included for analysis. A total of 1146 head-injured patients admitted to the Addenbrooke's Hospital Neurocritical Care Unit between 1992 and 2017 were included for the initial database. As described previously, this is likely to represent approximately $25 \%$ of all TBI admissions and $50 \%$ of all TBI admissions with ICP monitors [5]. Inclusion criteria were as follows: TBI; computerized invasive monitoring of ICP and $\mathrm{ABP}$ for at least $12 \mathrm{~h}$; admission Glasgow Coma Scale (GCS); and 6-month mortality data available. From this database of 1146 patients, files were selected that contained an initial ICP less than $25 \mathrm{~mm} \mathrm{Hg}$ with a subsequent ICP rise to over $40 \mathrm{~mm} \mathrm{Hg}$ for at least an hour. This yielded 33 suitable files. Patients were managed according to TBI guidelines [6] aimed at keeping ICP $<20 \mathrm{~mm}$ $\mathrm{Hg}$ and $\mathrm{CPP}>50-60 \mathrm{~mm} \mathrm{Hg}$. While all 33 patients in the cohort had computerized ICP monitoring, fewer patients had PRx monitoring $(n=24)$ or brain oxygenation monitoring $(n=9)$. This is because PRx monitoring only began in 1996 and brain oxygenation monitoring in 2004 .

\section{Data Acquisition and Processing}

ICP was monitored with an intraparenchymal sensor (Codman ICP MicroSensor, Codman \& Shurtleff, Raynham, Massachusetts). ABP (Baxter Healthcare, Deerfield, Illinois) at the level of the right atrium (1992-2015) and at the foramen of Monro (2015-2017-one patient). Brain tissue oxygenation was monitored using a Licox probe via a cranial access device (Technicam, Abbott, UK). Probes were positioned at a constant depth in the white matter, pericontusional in focal injuries or in the non-dominant frontal lobe in diffuse injuries. Probe positioning was verified by means of a head computed tomography $(\mathrm{CT})$ scan.

All data were sampled at least $50 \mathrm{~Hz}$ with proprietary data acquisition and analysis software (ICM 1992-2002 [7] and then with $\mathrm{ICM}+\odot$, http://www.neurosurg.cam. ac.uk/icmplus after 2002). Heart rate was determined as the fundamental frequency of the ABP signal over a $10 \mathrm{~s}$ window within the cardiac $(40-180$ cycles/min) frequency band. Amplitude of the cardiac pulse in ICP and $\mathrm{ABP}$ was determined as the fundamental amplitude in the cardiac frequency band (40-180 cycles/min). ABP and ICP signals were averaged (mean) over a 10-s window; then PRx was calculated as the moving Pearson correlation of 30 consecutive $\mathrm{ABP}$ and ICP, updated every minute. Cerebrospinal compensatory reserve (RAP) was calculated similarly as the moving correlation between mean ICP and the pulse amplitude of ICP. AMP was divided by aABP (giving AMP/aABP ratio) to get an indication of the transmission of the cardiac pulse from the blood pressure to the ICP.

ICP, ICP pulse amplitude, ABP, ABP pulse amplitude, and RAP data were available for all 33 patients, while PRx and $\mathrm{P}_{\mathrm{BT}} \mathrm{O}_{2}$ were available for 24 and 9 patients, respectively.

\section{Statistical Analysis}

Data are reported as means and standard deviations. The relationship between minute-by-minute values of mean ICP and ICP pulse amplitude was fitted with a generalized additive model allowing for three different segments (cubic regression spline smooth). The number of segments (3) was chosen a priori to allow for 3 different portions of the ICP, AMP relationship $(\sim$ flat at low ICP, steep rising segment, and an upper breakpoint).

For the aggregate relationship between ICP or CPP and intracranial parameters (PRx, RAP, AMP), the means of each variable from each patient were calculated in $10 \mathrm{~mm} \mathrm{Hg}$ wide intervals of ICP and CPP and then local 
regression smoothing (LOWESS) was applied. This binning procedure, prior to LOWESS fitting was to ensure that patients with longer recordings did not contribute disproportionately to parts of the smooth. Pairwise comparisons between physiological variables at the different ICP levels were performed using Student's $t$ test. No adjustments for multiple comparisons were made in this exploratory analysis. To test whether early PRx was different between those who developed refractory high ICP and TBI patients who did not, we first identified controls for the 24 cases who had simultaneous ICP and PRx data with 24 controls (selected from the 1146 patients) who were matched for sex, initial GCS and age. Then we performed a Wilcox test for the ICP values between the two groups. Because the primary aim of this study was descriptive, we used all available cases in the database rather than performing a priori sample size calculations. We used the $\mathrm{R}$ language and software environment for statistical computation (R Core Team 2015 version 2.12.1 [8]) using the following packages: dplyr [9], ggplot2 [10], gam [11], and MatchIt [12]. The significance level was set at 0.05 .

\section{Results}

Admission characteristics are displayed in Table 1. Of the 33 patients, 7 were female and the mean age was 30.3 . In the cohort, ICP rose from a mean minimum of 5.46 $( \pm 6.1)$ to a mean maximum of $74.67( \pm 22.76)$. Seventeen of the patients died.

Two patient examples illustrating the heterogeneous nature of the response to intracranial hypertension are depicted in Fig. 1. In the patient in Fig. 1a, PRx is initially preserved and only becomes impaired after the development of raised ICP. In the other patient (Fig. 1b), PRx is clearly disturbed prior to the development of severe refractory raised ICP. In this case, a decrease in $\mathrm{P}_{\mathrm{BT}} \mathrm{O}_{2}$ followed the decrease in CPP almost linearly to reach oxygen pressures of less than $5 \mathrm{~mm} \mathrm{Hg}$. In addition, a dissociation between the rise in mean ICP and pulse amplitude of ICP can be seen near the end of the recording, such that with increasing ICP, pulse amplitude remained constant. This failure for ICP pulse amplitude to increase despite increases in mean ICP has been postulated to be due to critical cerebrovascular collapse.

Across all patients, PRx (Fig. 2) showed a general increasing trend, signifying disturbed autoregulation with increasing ICP and of note, the PRx at baseline levels of ICP is disturbed in a number of the patients ('Appendix' Fig. 7). As expected, PRx plotted against CPP reveals a steadily increasing PRx with decreasing CPP. The relationship between mean ICP (and CPP) levels and ICP pulse amplitude is depicted in
Table 1 Patient demographics; cerebral effects of refractory intracranial hypertension after TBI

\begin{tabular}{ll} 
& Overall \\
\hline$N$ & 33 \\
Age (years) (mean (sd)) & $30.30(12.54)$ \\
Sex=male (number, \%) & $26(78.8)$ \\
GCS $<=8$ (number, \%) & $28(84.8)$ \\
Monitoring length (h) (mean (sd)) & $188.53(145.40)$ \\
Decompressive craniectomy (number, \%) & \\
No & $15(45.5)$ \\
Yes & $11(33.3)$ \\
NA & $7(21.2)$ \\
ICP (mm Hg) (mean (sd)) & $25.60(8.71)$ \\
Max ICP (mm Hg) (mean (sd)) & $74.67(22.76)$ \\
Min ICP (mm Hg) (mean (sd)) & $5.46(6.10)$ \\
CPP (mm Hg) (mean (sd)) & $67.70(10.98)$ \\
PRx (a.u.) (mean (sd); $n=24)$ & $0.16(0.25)$ \\
Mortality=dead (\%) & $17(51.5)$ \\
\hline CPP cerebral perfusion pressure
\end{tabular}

CPP cerebral perfusion pressure, GCS Glasgow coma scale, ICP intracranial pressure, $P R x$ pressure reactivity index

Figs. 3 and 4. In general, ICP amplitude increased monotonically with increasing mean ICP (12 patients; Fig. 3c); however, in 6 of the cases an upper breakpoint (a switch from a positive to a negative relationship between mean ICP and ICP pulse amplitude) is seen at high ICPs (Fig. 3a). In 13 patients, a rightward deflection of the AMP-ICP was detected (Fig. 3b). In the remaining 2 patients, AMP monotonically decreased with increasing ICP (up until ICP $40 \mathrm{~mm} \mathrm{Hg}$-not included in figure). Similar responses were seen when ICP pulse amplitude was normalized to the arterial pulse amplitude (Fig. 4, middle panel). The index of cerebral compensatory reserve, RAP, increased from low to moderate levels of ICP and thereafter showed a gradual decline with further increases in ICP (Fig. 4e). At all levels of ICP or CPP, the averaged RAP response was greater than +0.3 a.u.

When all 9 available $\mathrm{P}_{\mathrm{BT}} \mathrm{O}_{2}$ responses are viewed together (Fig. 5), $\mathrm{P}_{\mathrm{BT}} \mathrm{O}_{2}$ shows a steady decrease with increasing ICP. However, the between patients response is strikingly variable (Fig. 8 'Appendix'). When $\mathrm{P}_{\mathrm{BT}} \mathrm{O}_{2}$ is plotted against CPP, a consistent pattern is seen, with a relatively preserved $\mathrm{P}_{\mathrm{BT}} \mathrm{O}_{2}$ with mild decreases in CPP followed by a steeper decrease with moderate to severe decreases in CPP. All but one patient shows a decrease in $\mathrm{P}_{\mathrm{BT}} \mathrm{O}_{2}$ with decreasing CPP.

When compared to 24 controls (severe TBI patients, matched for age, sex, and initial GCS), initial mean PRx in the first five hours of monitoring was higher (Fig. 6) in the cases of refractory intracranial hypertension. 
A
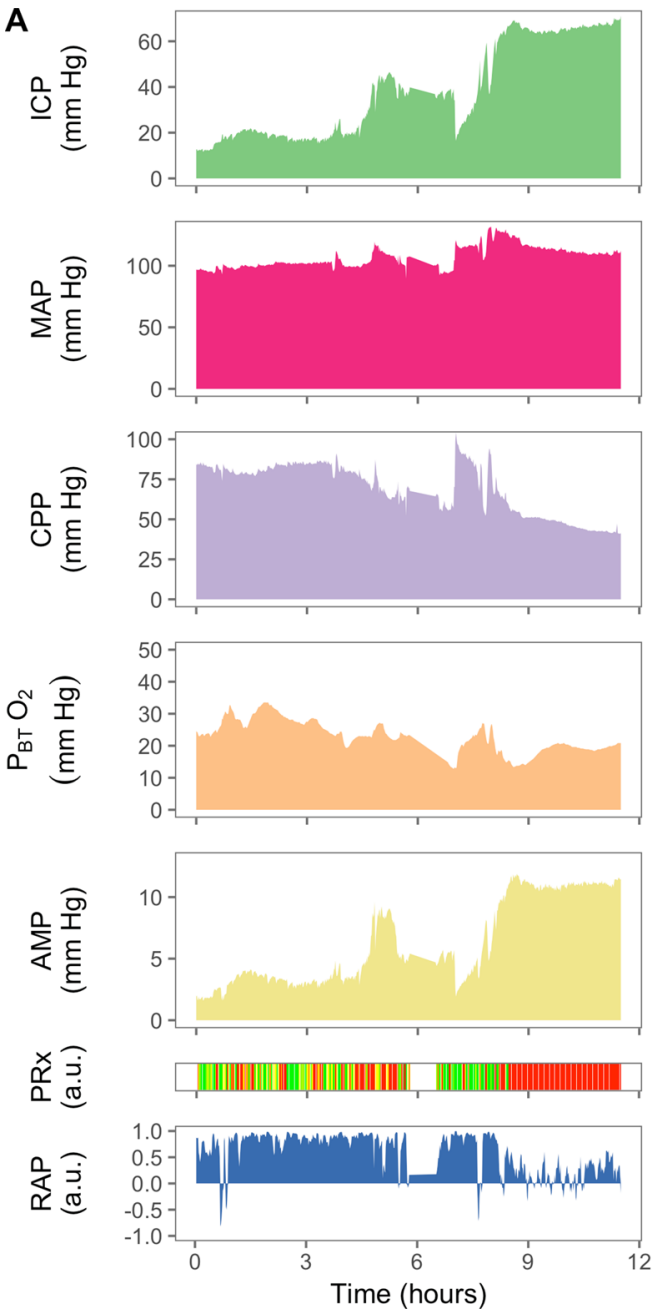

B
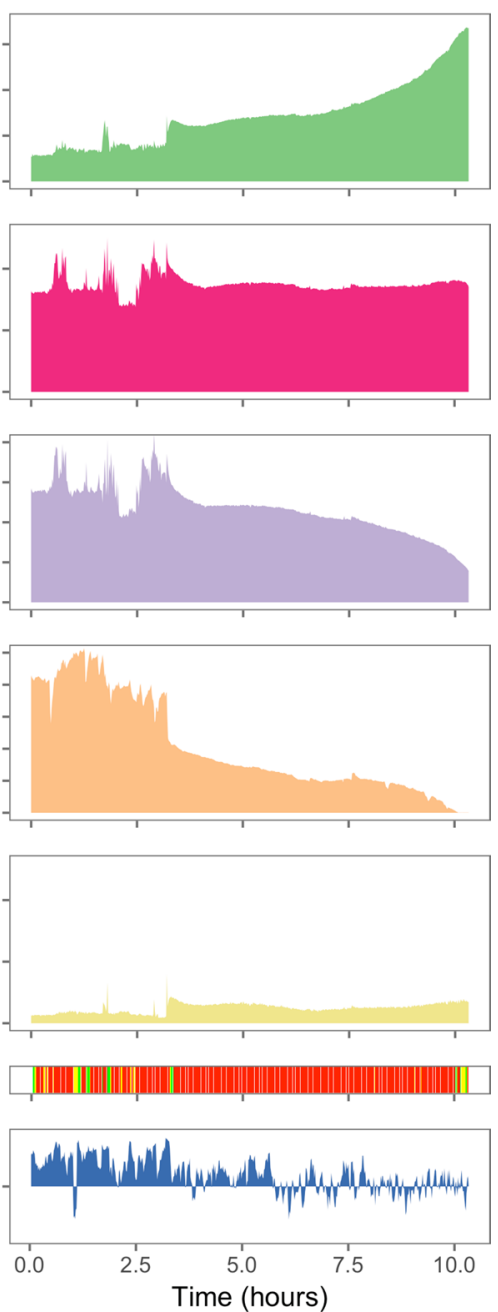

Fig. 1 Neuromonitoring during severe intracranial hypertension in two traumatic brain-injured patients. On the left, ICP rises to $70 \mathrm{~mm} \mathrm{Hg}$ over a period of $12 \mathrm{~h}$ and PRx was not significantly impaired prior to the increase in mean ICP. In addition, although $\mathrm{P}_{B T} \mathrm{O}_{2}$ decreased with increasing ICP, it did not reach severely hypoxic values even during the maximal mean ICP $\left(\mathrm{P}_{\mathrm{BT}} \mathrm{O}_{2} \sim 20 \mathrm{~mm} \mathrm{Hg}\right.$ at ICP $\left.60 \mathrm{~mm} \mathrm{Hg}\right)$. On the right, ICP rises dramatically from below $20 \mathrm{~mm} \mathrm{Hg}$ to over $60 \mathrm{~mm} \mathrm{Hg}$ in the space of $10 \mathrm{~h}$. This increase in ICP was associated with a fall in CPP, and brain tissue oxygenation. In this case, PRx was disturbed (>0.25) even in the first $3 \mathrm{~h}$, while ICP was under $20 \mathrm{~mm}$ Hg. Despite large increases in ICP over the last $5 \mathrm{~h}$, pulse amplitude of ICP (yellow) shows little change. These two cases illustrate that refractory intracranial hypertension may have different neuromonitoring phenotypes. ICP intracranial pressure; AMP pulse amplitude of ICP; MAP mean arterial pressure; CPP cerebral perfusion pressure; $\mathrm{P}_{\mathrm{BT}} \mathrm{O}_{2}$ brain tissue oxygenation; $\mathrm{PRx}$ pressure reactivity index; RAP index of cerebrospinal compensatory reserve

While ICP was also higher, this did not reach statistical significance (Wilcox $p=0.197$ ).

Grouping each of the patients' data into three ICP groups $(<25,25-50,>50 \mathrm{~mm} \mathrm{Hg}$; Table 2) confirmed the visual interpretation of Figs. 2, 3, and 4).

\section{Discussion}

By isolating the rare cases of severe refractory intracranial hypertension with multimodality monitoring, we describe the cerebral physiological response to raised ICP after TBI. Although only exploratory, these data highlight the impact of raised ICP on cerebral autoregulation, the sensitivity of brain tissue oxygenation to raised ICP and the possible role of impaired pressure reactivity in identifying at-risk patients.

\section{Autoregulation Parameters}

Raised ICP impairs dynamic cerebral autoregulation (Fig. 2, Table 2). This confirms previous investigations of autoregulation during short-term increases in ICP (plateau waves) $[13,14]$ and is consistent with a recent large between patient analysis that found a significant 


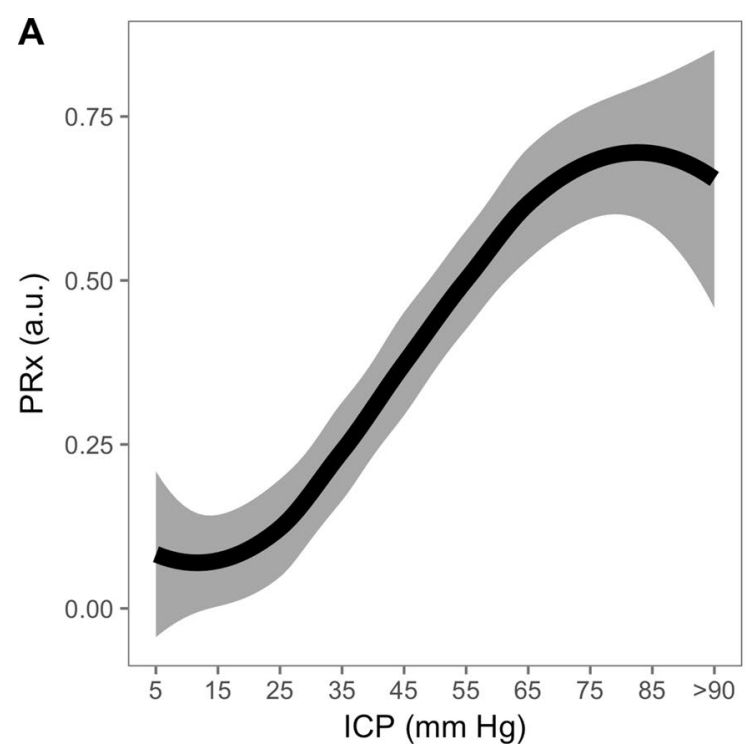

B

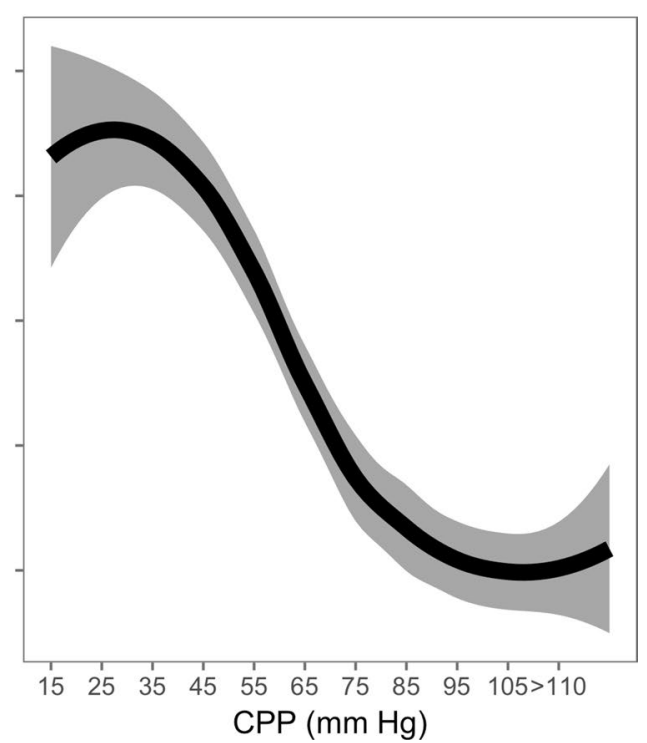

Fig. 2 PRx response to refractory intracranial hypertension expressed relative to changes in ICP (left) and CPP (right) (LOWESS with 95\% confidence interval; $n=24)$. Pressure reactivity increased with increasing ICP and PRx plotted against CPP revealed a partial 'U-shaped' curve as previously described. PRx is well maintained until CPP drops below $70 \mathrm{~mm} \mathrm{Hg}$, below which PRx deteriorates. PRx pressure reactivity; ICP intracranial pressure; CPP cerebral perfusion pressure

correlation between mean ICP over the whole monitoring period and mean PRx [15]. In contrast to these studies, however, the current analysis observed changes within patients and over periods of time that are substantially longer than the calculation window for PRx. Therefore, the finding of impaired PRx with increasing ICP is unlikely to be explained by between patient confounding factors or time-resolution limitations of the PRx method. Similarly, PRx deteriorates with decreased CPP, representing the lower half of the 'U-shaped' relationship between CPP and PRx [16].

Perhaps most striking though, is that PRx was disturbed before the onset of intracranial hypertension in a selection of the patients. This has been observed previously in a cohort of brain-injured patients of mixed pathologies (subarachnoid hemorrhage, hypoxic brain injury, trauma), but has not been statistically assessed [17]. After matching cases of severe refractory high ICP for age, initial GCS, and sex, we found that PRx in the first $5 \mathrm{~h}$ was higher in those who developed raised ICP. The same was not true for the mean ICP over the first $5 \mathrm{~h}$, although this did approach statistical significance $(p=0.197)$. This highlights the potential utility of continuous assessments of cerebral autoregulation in predicting raised ICP events. Encouragingly, a previous investigation found that a feature similar to PRx (the long term mean arterial pressure (MAP)-ICP correlation from low-frequency data) can help predict periods of raised ICP $30 \mathrm{~min}$ before they occur [18]. Identifying patients at risk of raised ICP may prove crucial as raised ICP carries a high risk of mortality, and perhaps the most effective ICP treatments, such as decompression, require some time to mobilize.

Reasons why impaired cerebral pressure reactivity may be a harbinger of raised ICP in some patients but not others deserves further discussion as it perhaps reflects different pathophysiological profiles of intracranial hypertension. Intracranial hypertension is a result of changes in compartmental volumes within the brain: increases in volume of the brain tissue (for example cerebral edema), the cerebral spinal fluid, or the blood (venous or arterial). Disturbed autoregulation preceding an increase in ICP may indicate a primary vascular dysfunction that, over time, causes raised ICP. In these cases, a vascular dysfunction as manifest in impaired PRx could lead to vasogenic edema, which over hours could lead to progressive increases in ICP. Cases where ICP and PRx are disturbed simultaneously indicate that either the impaired vascular function is a consequence of the raised ICP, or the underlying pathophysiological mechanism is occurring rapidly. Simultaneous perturbations in PRx and ICP could conceivably occur with obstruction in CSF flow, a rapidly expanding lesion or during a sudden increase in blood volume. 

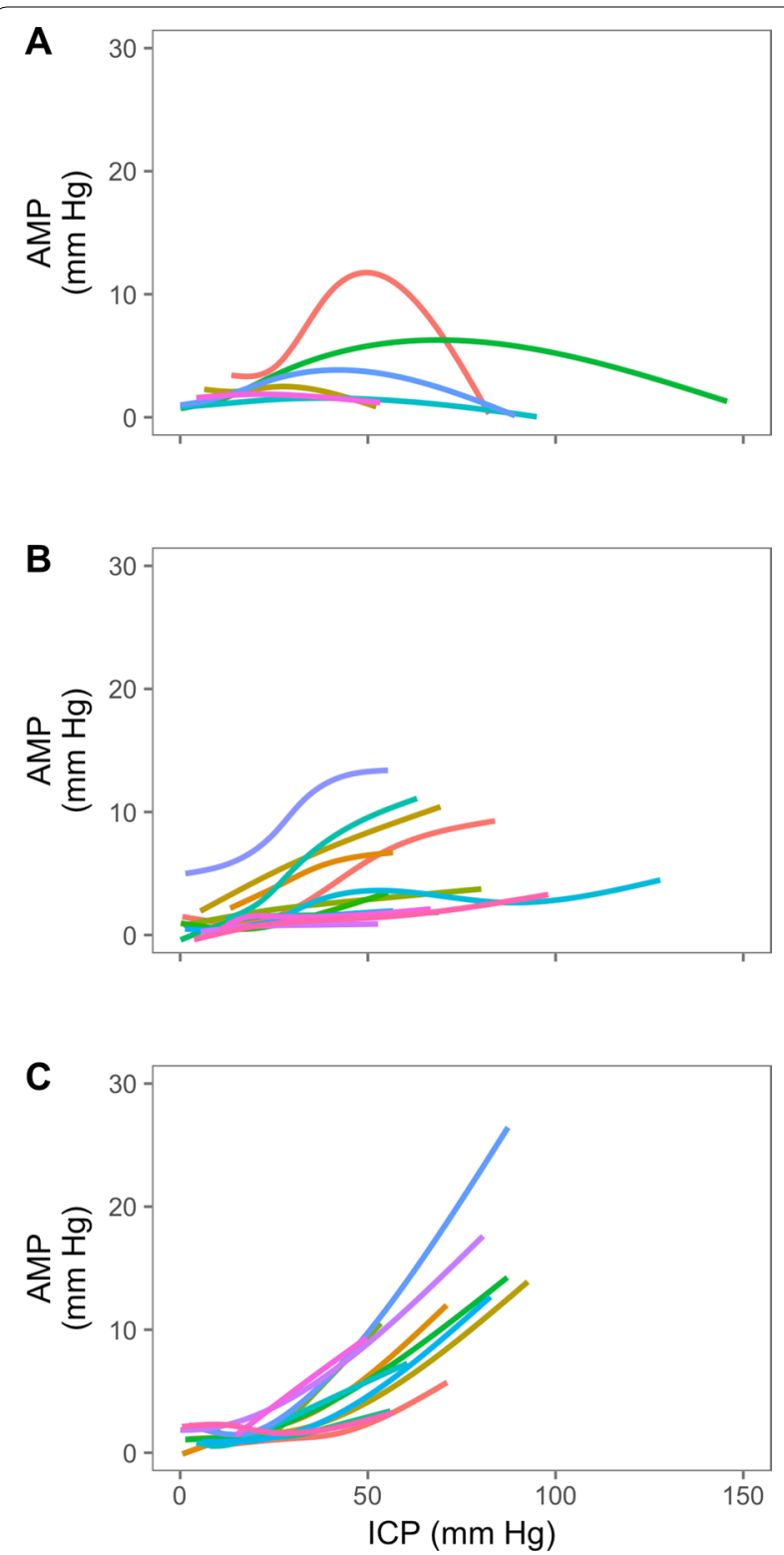

Fig. 3 The ICP amplitude - mean ICP relationship $(n=33)$. Three distinct patterns were identified; those with an upper breakpoint ( $A$, $n=6)$, a rightward deflected pattern $(B, n=13)$ or those with a monotonic increasing pattern $(C, n=12)$. In 2 patients, AMP decreased with increasing ICP (not shown). ICP intracranial pressure; AMP pulse amplitude of ICP

In addition, we found a consistent increase in MAP at elevated levels of ICP (Table 1. While previous experimental work has shown profound Cushing vasopressor responses to extreme and rapid increases in ICP $[2,19]$, in these data we cannot rule out a confounding effect of concurrent interventions and medications such as cooling or vasopressors.

\section{Brain Oxygenation}

In this sample of patients with extreme increases in ICP, $\mathrm{P}_{\mathrm{BT}} \mathrm{O}_{2}$ overall decreased. This is consistent with a recent experimental study which demonstrated a cortical vulnerability to increased ICP [4]. However, there was marked variation between patients in the levels of $\mathrm{P}_{\mathrm{BT}} \mathrm{O}_{2}$ and the response to increased ICP. This highlights the complex nature of the $\mathrm{P}_{\mathrm{BT}} \mathrm{O}_{2}$ variable. It does not merely index brain perfusion, but the complicated interplay between cerebral oxygen delivery (dependent on $\mathrm{P}_{\mathrm{a}} \mathrm{O}_{2}$, $\mathrm{Hb}, \mathrm{CBF})$, metabolic rate of the nearby cerebral tissues, and any diffusion barriers [20-22]. Therefore, many clinical scenarios, potentially independent of perfusion, may affect brain oxygenation such as red blood cell transfusion, hypoxia or hyperoxia, mitochondrial dysfunction [23-25]. The position of the oxygen sensing probe may also be relevant as intracontusional oxygen monitoring should be different to pericontusional or healthy tissue monitoring [26]. Furthermore, the depth of probe may also be relevant; in basilar artery dependent rabbits with CSF infusion induced increases in ICP, the cortical laser Doppler flux was more sensitive to increases in ICP than the global basilar artery flow velocity [4].

Nevertheless, monitoring of brain oxygenation, sometimes in combination with cerebral microdialysis has increased in popularity over last decade with some promising initial results. In a retrospective analysis comparing before $\mathrm{P}_{\mathrm{BT}} \mathrm{O}_{2}$ to after $\mathrm{P}_{\mathrm{BT}} \mathrm{O}_{2}$-targeted therapy, found that keeping $\mathrm{P}_{\mathrm{BT}} \mathrm{O}_{2}$ above $20 \mathrm{~mm} \mathrm{Hg}$ was associated with decreased mortality [27]. In addition, similar to $\mathrm{PRx}$, brain tissue monitoring has been combined with $\mathrm{ABP}$ and CPP monitoring to yield continuous autoregulation assessment in TBI and subarachnoid hemorrhage [28-30].

\section{The Mean ICP and ICP Pulsatile Amplitude Relationship}

The increase in pulsatility of ICP with increasing levels of mean ICP has been interpreted as a loss of cerebral compensatory reserve. In this scenario, when the intracranial system is on the steep ascending portion of the pressure-volume curve, a pulsatile injection of blood volume from the cardiac cycle would be expected to produce a large increase in pulsatile pressure. However, at extreme levels of ICP (approaching diastolic blood pressure) it has been demonstrated in animal models that ICP pulsations may in fact decrease [31]. This has been proposed to be related to critical closing of the 


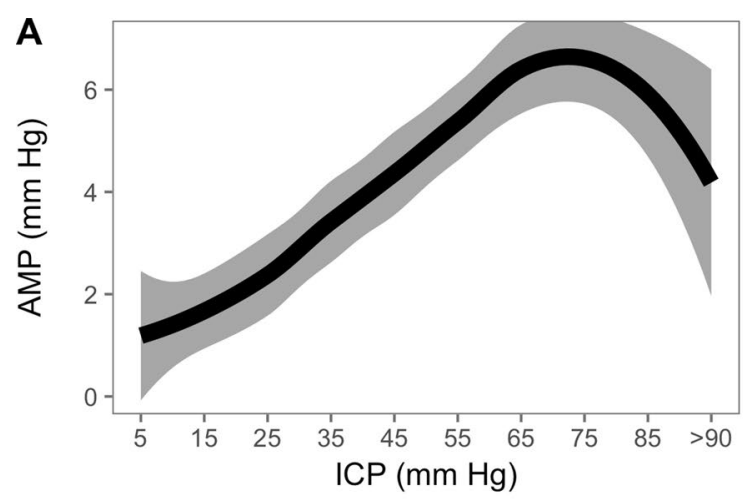

B

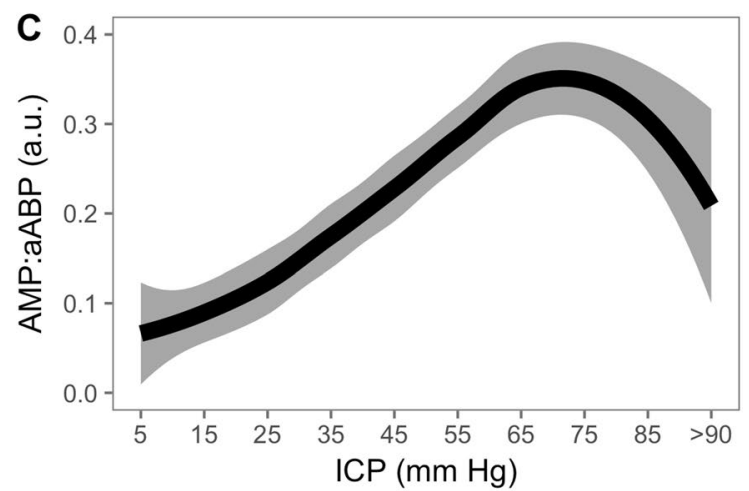

D
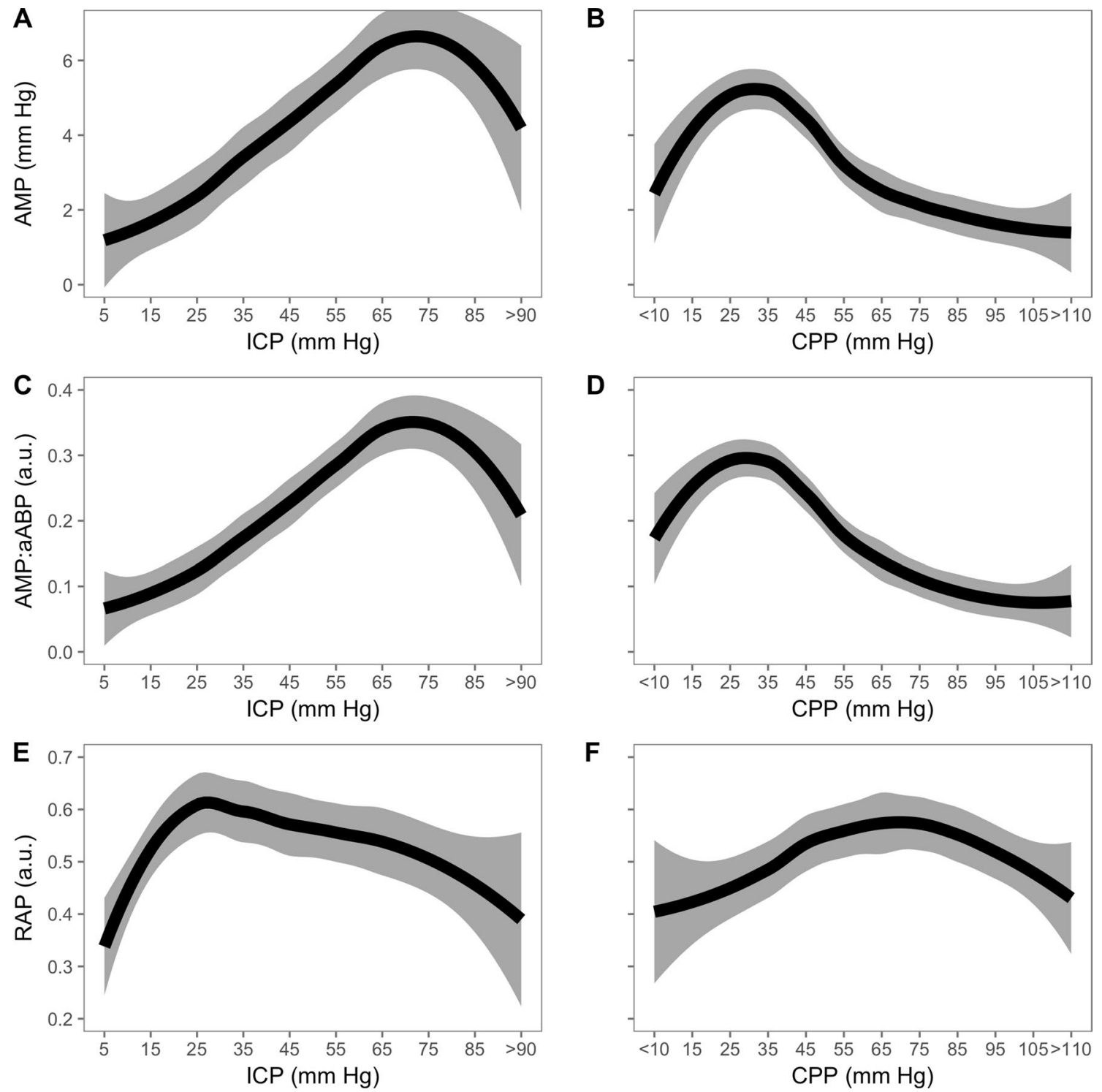

$\mathbf{F}$

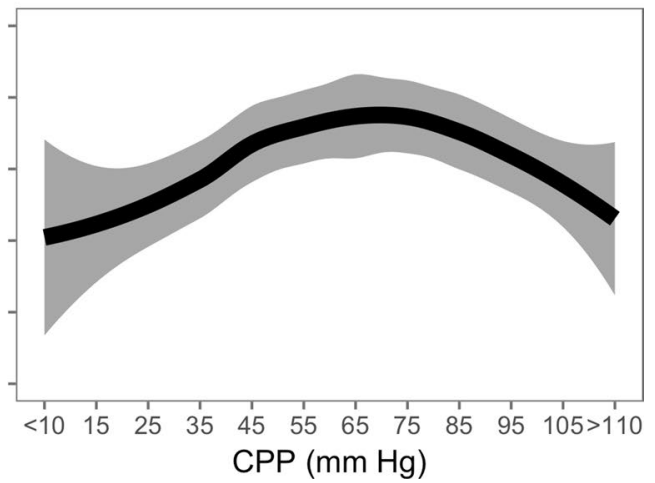

Fig. 4 Relationship between ICP (left) or CPP (right) with ICP amplitude, transmission of arterial to intracranial pulse, and RAP (LOWESS with 95\% confidence interval; $n=33$ ). When all patients are grouped together, an upper breakpoint in the AMP-ICP relationship occurs at around $70 \mathrm{~mm} \mathrm{Hg}$. A similar response is seen for arterial to intracranial pulse transmission indicating that a decreased ABP amplitude is not responsible for the AMP-ICP upper breakpoint. RAP increases from low $(0 \mathrm{~mm} \mathrm{Hg})$ to moderate ICP $(\sim 30 \mathrm{~mm} \mathrm{Hg})$ and thereafter decreases with further increase in ICP. ICP intracranial pressure; CPP cerebral perfusion pressure; RAP cerebrospinal compensatory reserve

cerebrovascular bed [7]. Observing this phenomenon is, however, far from universal in refractory intracranial hypertension (Fig. 3), probably as its occurrence is multifactorial, depending on parameters such as vasomotor tone, cerebral intravascular pressures and arterial blood pulse pressure. Interestingly, RAP-the short-term correlation between changes in mean ICP and mean amplitude of ICP-did not show a similar upper breakpoint like the pulsatility-mean ICP relationship and rarely reached negative values (Fig. 4 and 1) as may have been expected at these extreme levels of ICP [32]. This perhaps reflects the short-term dynamic nature of the RAP calculation (calculated over a 5-min time window) compared with the extended time windows associated with the pulsatility-mean ICP relationship.

\section{Limitations}

Due to the small sample size, these analyses must be considered as preliminary descriptions. While it was possible to include more patients by relaxing the definition 

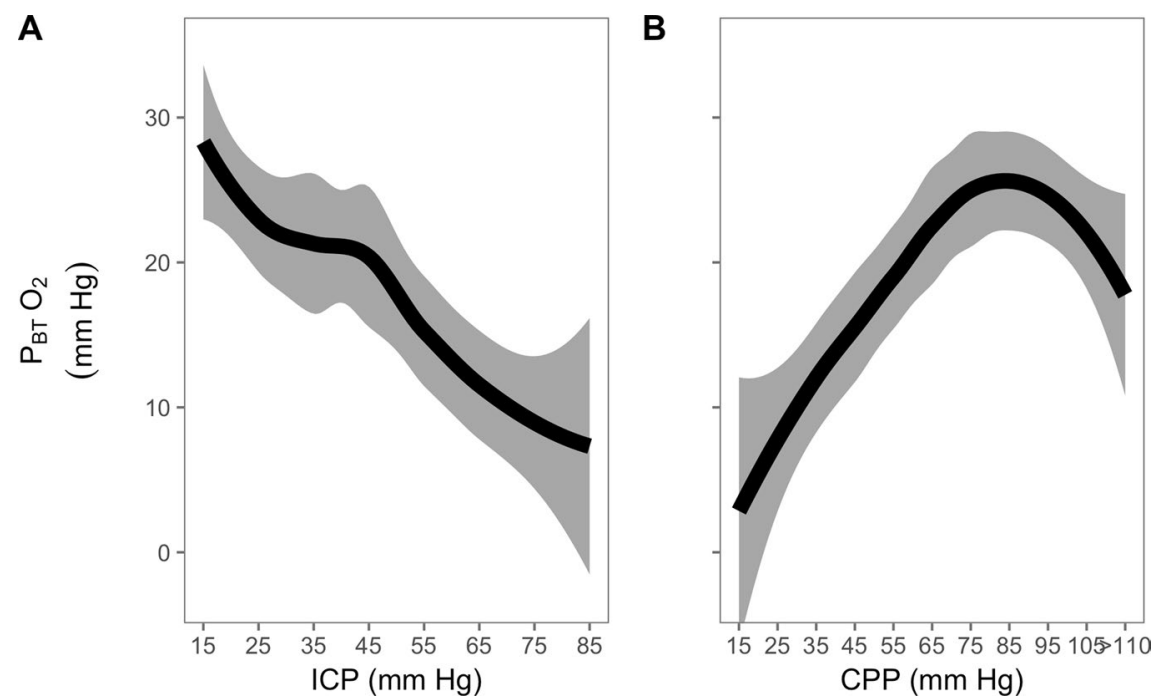

Fig. $5 \mathrm{P}_{\mathrm{BT}} \mathrm{O}_{2}$ response to refractory intracranial hypertension expressed relative to changes in ICP (left) and CPP (right) (LOWESS with 95\% confidence interval; $n=9)$. When expressed against ICP, $\mathrm{P}_{\mathrm{BT}} \mathrm{O}_{2}$ demonstrates a steady decrease. When expressed in relation to changes in CPP, the relationship resembles the autoregulation curve; with moderate levels of CPP (70-> $90 \mathrm{~mm} \mathrm{Hg}$ ), oxygenation is well maintained, but lower than $70 \mathrm{~mm}$ Hg, oxygenation decreases (by approximately $0.5 \mathrm{~mm} \mathrm{Hg}$ per $1 \mathrm{~mm} \mathrm{Hg}$ decrease in CPP. $\mathrm{P}_{\mathrm{BT}} \mathrm{O}_{2}$ brain tissue oxygenation; ICP intracranial pressure; CPP cerebral perfusion pressure

of refractory intracranial hypertension, we wished to describe the physiological response across the widest range of ICPs and therefore opted to only include patients with severe intracranial hypertension. Further, because intracranial physiology will depend heavily on concurrent therapies, detailed clinical annotations to the monitoring data would aid interpretation and increase the generalizability of the study. Stratification of physiological responses to intracranial hypertension by ICP treatment modality (for example decompression or barbiturates) may yield useful information describing early indicators of a beneficial versus a pathological physiological response. The association between early PRx and later development of intracranial hypertension needs to be treated with caution as the case-control analysis was only carried out in a small subset of patients with concurrent PRx monitoring $(n=24)$. Furthermore, time points besides the first $5 \mathrm{~h}$ for the ability of PRx to predict intracranial hypertension should be investigated. In addition, the current study does not address the pragmatic issue of how multimodality monitoring data can be integrated into clinical care without causing a detrimental information overload. While ongoing data collection with the increasing availability of multimodality monitoring will likely shed light on this area in the near future, controlled experimental studies that mimic the increase

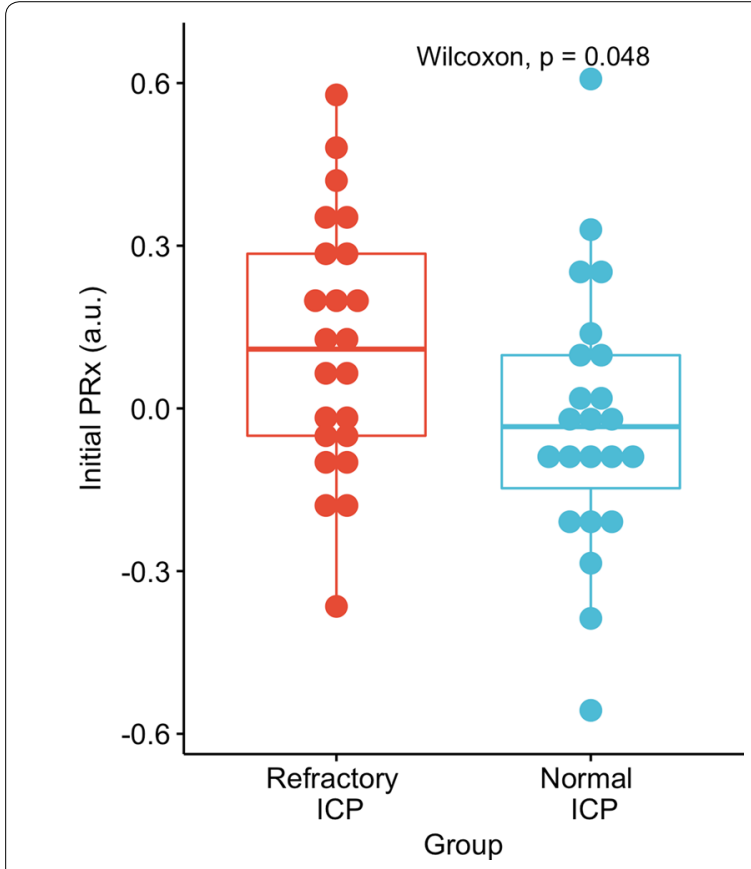

Fig. 6 PRx over the first $5 \mathrm{~h}$ in patients who went on to develop severe refractory intracranial hypertension $(n=24)$ compared to severe TBI patients matched for age, sex and initial GCS $(n=24)$.

Those who developed severe refractory intracranial hypertension tended to have higher PRx in the first $5 \mathrm{~h}$ of monitoring $(p=0.048)$ while ICP was not significantly different between the two groups $(p=0.197)$. PRx pressure reactivity index; ICP intracranial pressure 
Table 2 Physiologic response to high intracranial pressure after TBI (mean (sd); $n=33$ unless stated otherwise)

\begin{tabular}{|c|c|c|c|c|c|}
\hline Variable & $0-25 \mathrm{~mm} \mathrm{Hg}$ & $25-50 \mathrm{~mm} \mathrm{Hg}$ & $50-150 \mathrm{~mm} \mathrm{Hg}$ & $\begin{array}{l}p \text { (elevated vs. } \\
\text { base) }\end{array}$ & $\begin{array}{l}p \text { (severe vs } \\
\text { elevated) }\end{array}$ \\
\hline ICP $(\mathrm{mm} \mathrm{Hg})$ & $17.52(3.85)$ & $32.80(2.39)$ & $61.04(10.79)$ & $<0.001$ & $<0.001$ \\
\hline AMP (mm Hg) & $1.83(1.36)$ & $3.10(2.08)$ & $5.34(4.26)$ & 0.02 & $<0.001$ \\
\hline AMP/aABP (a.u.) & $0.10(0.06)$ & $0.17(0.10)$ & $0.29(0.22)$ & 0.02 & $<0.001$ \\
\hline CPP $(\mathrm{mm} \mathrm{Hg})$ & $73.90(9.27)$ & $63.79(10.41)$ & $39.27(18.11)$ & $<0.001$ & $<0.001$ \\
\hline MAP (mm Hg) & $91.42(9.87)$ & $96.61(9.48)$ & $99.82(12.55)$ & 0.01 & 0.120 \\
\hline $\mathrm{aABP}(\mathrm{mm} \mathrm{Hg})$ & $18.87(3.32)$ & $19.70(4.21)$ & $19.85(5.85)$ & 0.16 & 0.712 \\
\hline $\mathrm{HR}$ (bpm) & $77.53(15.35)$ & $79.46(19.04)$ & $83.78(20.72)$ & 0.50 & 0.214 \\
\hline $\begin{array}{l}\operatorname{PRx}(\text { a.u. }) \\
(n=24)\end{array}$ & $0.06(0.26)$ & $0.21(0.30)$ & $0.57(0.24)$ & 0.01 & $<0.001$ \\
\hline RAP (a.u.) & $0.54(0.20)$ & $0.59(0.25)$ & $0.46(0.27)$ & 0.29 & 0.005 \\
\hline $\mathrm{P}_{\mathrm{BT}} \mathrm{O}_{2}(\mathrm{~mm} \mathrm{Hg})(n=9)$ & $27.27(7.32)$ & $20.78(5.43)$ & $12.68(7.09)$ & 0.06 & 0.02 \\
\hline
\end{tabular}

$I C P$ intracranial pressure, $A M P$ pulse amplitude of ICP, $a A B P$ amplitude of arterial blood pressure, $M A P$ mean arterial pressure, $C P P$ cerebral perfusion pressure, $H R$ heart rate, $P R x$ pressure reactivity index, $R A P$ cerebrospinal compensatory reserve, $P_{B T} O_{2}$ brain tissue oxygenation

in ICP observed after human TBI need to also play a role as they can effectively isolate the effects of the disease from that of treatment.

\section{Conclusion}

Severe intracranial hypertension after TBI leads to decreased brain oxygenation, impaired pressure reactivity, and a characteristic ICP pulsatility response.

\begin{abstract}
Author details
${ }^{1}$ Brain Physics Laboratory, Division of Neurosurgery, Department of Clinical Neurosciences, Cambridge Biomedical Campus, University of Cambridge, Cambridge, UK. ${ }^{2}$ Department of Anaesthesiology, Level 12 Auckland Support Building, Auckland City Hospital, University of Auckland, 2 Park Road, Grafton, Auckland, New Zealand. ${ }^{3}$ Division of Neurosurgery, Department of Clinical Neurosciences, Cambridge Biomedical Campus, University of Cambridge, Cambridge, UK. ${ }^{4}$ Division of Anaesthesia, Department of Medicine, Addenbrooke's Hospital, University of Cambridge, Cambridge, UK. ${ }^{5}$ Department of Surgery, Rady Faculty of Health Sciences, University of Manitoba, Winnipeg, Canada. ${ }^{6}$ Biomedical Engineering, Faculty of Engineering, University of Manitoba, Winnipeg, Canada. ${ }^{7}$ Department of Human Anatomy and Cell Sciences, Rady Faculty of Health Sciences, University of Manitoba, Winnipeg, Canada. ${ }^{8}$ Department of Anesthesiology, Pharmacology and Therapeutics, Vancouver General Hospital, University of British Columbia, Vancouver, Canada. ${ }^{9}$ NIHR Global Health Research Group on Neurotrauma, University of Cambridge, Cambridge, UK. ${ }^{10}$ Institute of Electronic Systems, Warsaw University of Technology, Warsaw, Poland.
\end{abstract}

\section{Author Contributions}

The study concept and design were given by JD, PS, PH, DKM, and MC. Data acquisition was performed by JD, PS, HA, FAZ, DC, XL, and MC. Data analysis was performed by JD, FAZ, MF, and MC. JD prepared the first draft of the manuscript. Interpretation of the data was done by PS, PH, DKM, MF, and MC. All authors provided critical feedback of the manuscript and approved the final version.

\section{Source of Support}

This study was supported by National Institute for Healthcare Research (NIHR, UK) through the Acute Brain Injury and Repair theme of the Cambridge NIHR Biomedical Research Centre, an NIHR Senior Investigator Award to Dr Menon, and an NIHR Research Professorship to Dr Hutchinson.

\section{Conflicts of interest}

These studies were supported by National Institute for Healthcare Research (NIHR, UK) through the Acute Brain Injury and Repair theme of the Cambridge NIHR Biomedical Research Centre, an NIHR Senior Investigator Award to Dr Menon, and an NIHR Research Professorship to Dr Hutchinson. Dr Donnelly is supported by a Woolf Fisher Scholarship (New Zealand). Dr Zeiler has received salary support for dedicated research time from the Cambridge Commonwealth Trust Scholarship, the University of Manitoba Clinician Investigator Program, and the Royal College of Surgeons of Canada-Harry S. Morton Travelling Fellowship in Surgery, during which this project was completed. Dr Hutchinson and Dr Menon were also supported by a European Union Framework Program 7 grant (CENTER-TBl; Grant Agreement No. 602150). Dr Zeiler and Dr Cardim have received PhD funding from the Cambridge Commonwealth Trust. Dr Hutchinson, and Dr Menon declares funding from the NIHR Global Health Research Group on Neurotrauma, which was commissioned by the NIHR using Official Development Assistance (ODA) funding (project 16/137/105). The views expressed in this publication are those of the author(s) and not necessarily those of the NHS, National Institute for Health Research or the Department of Health. The authors have the following potential conflicts of interest: Dr Czosnyka receives part of licensing fees for ICM+, owned and distributed by Cambridge Enterprise Ltd; paid lectures for Integra Lifescience Speakers'Bureau; and is an unpaid member of Board of Directors of Medicam Ltd, UK. Dr Smielewski receives part of the licensing fees for the software ICM (Cambridge Enterprise Ltd, Cambridge UK), used in this project for data collection and data preprocessing. The other authors have no personal, financial, or institutional interest in any of the drugs, materials, or devices described in this article. Dr Menon has had consultancy, research collaboration, or trial data monitoring interactions with Solvay Ltd; GlaxoSmithKline Ltd; Brainscope Ltd; Ornim Medical; Shire Medical and Neurovive Ltd; Calico Inc; Pfizer Ltd; Pressura Ltd; Glide Pharma Ltd; and NeuroPro Ltd.

\section{Ethical approval}

Monitoring of brain modalities was conducted as a part of standard patient care and archived in an anonymized database of physiological monitoring. No attempt was made to re-access clinical records for additional information. Since all data were extracted from the hospital records and fully anonymized, no data on patient identifiers were available, and therefore, formal patient or proxy consent and institutional ethics approval were not required.

\section{Open Access}

This article is distributed under the terms of the Creative Commons Attribution 4.0 International License (http://creativecommons.org/licenses/by/4.0/), which permits unrestricted use, distribution, and reproduction in any medium, provided you give appropriate credit to the original author(s) and the source, provide a link to the Creative Commons license, and indicate if changes were made. 


\section{Appendix}

See Figs. 7 and 8.
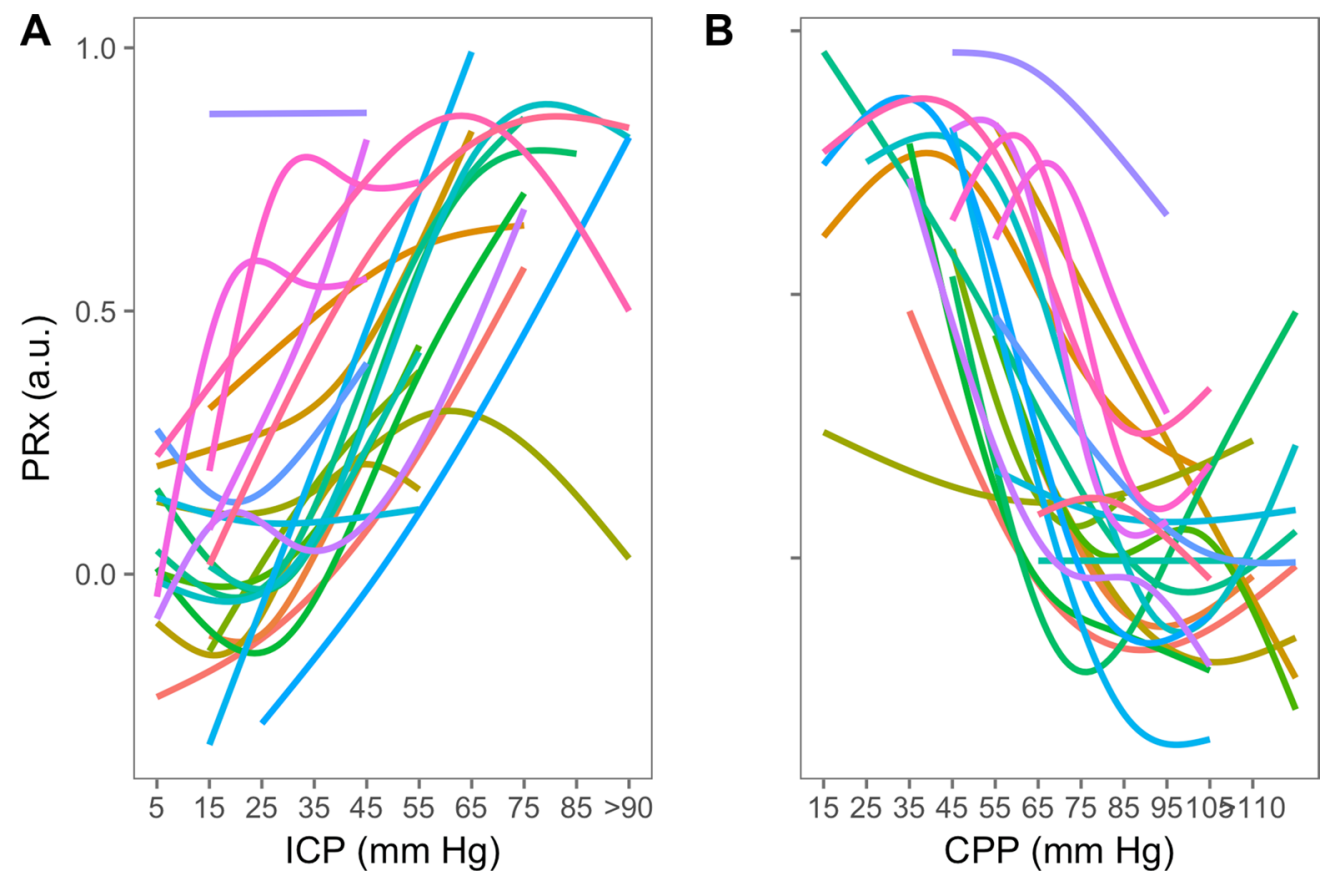

Fig. 7 Individual PRx responses to refractory intracranial hypertension expressed relative to changes in ICP (left) and CPP (right) $(n=24)$. While pressure reactivity index increased with increasing ICP in each patient, in some patients, PRx was disturbed (above $>0.25$ ) even at relatively normal ICP (below $25 \mathrm{~mm} \mathrm{Hg}$ ). PRx plotted against CPP revealed a partial 'U-shaped' curve in most patients, as previously described. PRx is well maintained until CPP drops below $70 \mathrm{~mm} \mathrm{Hg}$, below which PRx deteriorates. PRx pressure reactivity; ICP intracranial pressure; CPP cerebral perfusion pressure
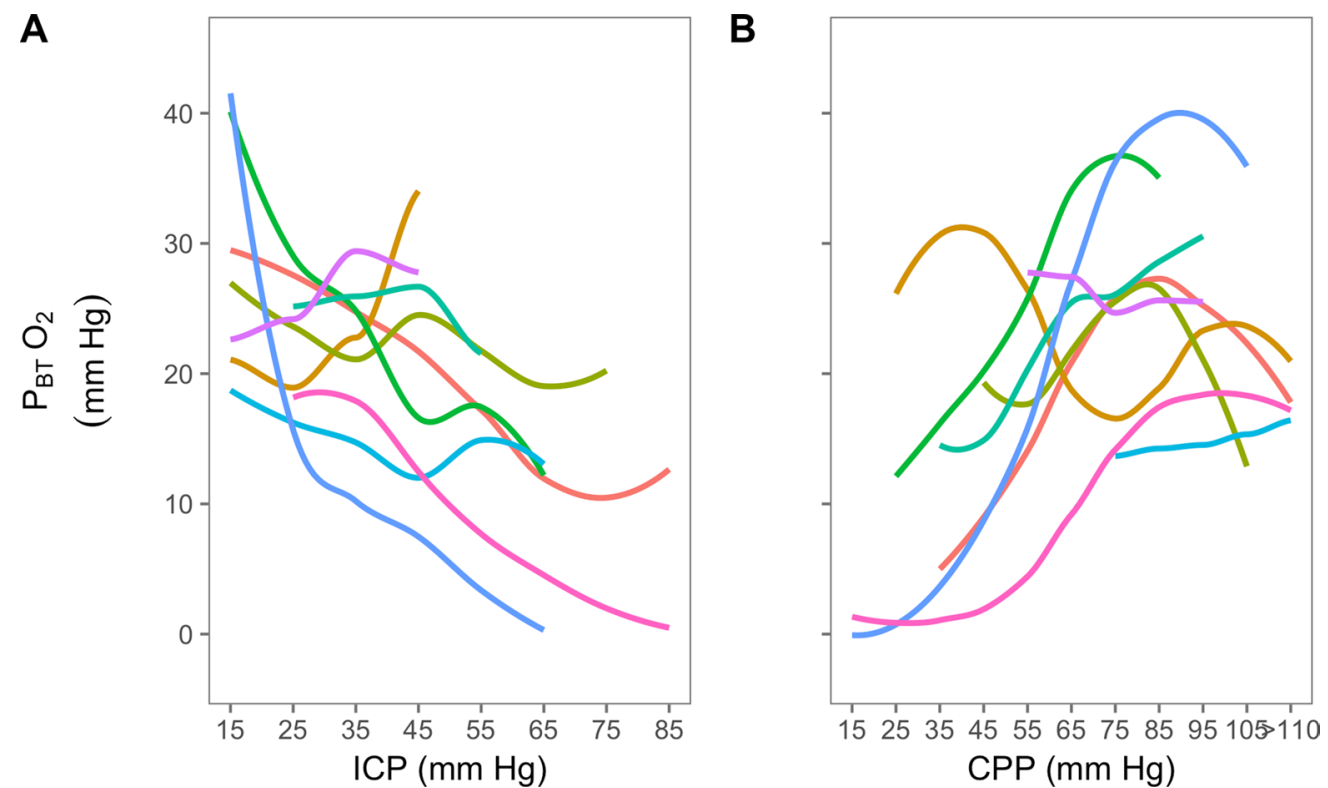

Fig. 8 Individual $\mathrm{P}_{\mathrm{B} T} \mathrm{O}_{2}$ response to refractory intracranial hypertension expressed relative to changes in ICP (left) and CPP (right) $(n=9)$. Brain tissue oxygenation response to increases in ICP showed marked variability between patients with some patients showing relatively preserved $\mathrm{P}_{\mathrm{B} T} \mathrm{O}_{2}$ with increasing ICP, some showing precipitous hypoxia, and some showing a paradoxical increase in oxygenation. A similar variability is observed with respect to CPP. This variability highlights the complex nature of the $\mathrm{P}_{\mathrm{BT}} \mathrm{O}_{2}$ signal; it is contributed to by respiratory $\left(\mathrm{FiO}_{2}, \mathrm{PaO}_{2}\right)$, hematological $(\mathrm{Hb})$, cardiovascular (cardiac output, cerebral blood flow) and metabolic factors (brain metabolism) 


\section{Publisher's Note}

Springer Nature remains neutral with regard to jurisdictional claims in published maps and institutional affiliations.

Published online: 25 June 2019

\section{References}

1. Stocchetti N, Maas AIR. Traumatic intracranial hypertension. N Engl J Med. 2014;370:2121-30.

2. Nagao $S$, Sunami $N$, Tsutsui T, Honma Y, Momma F, Nishiura T, et al. Acute intracranial hypertension and brain-stem blood flow. An experimental study. J Neurosurg. 1984;60:566-71.

3. Nakagawa Y, Tsuru M, Yada K. Site and mechanism for compression of the venous system during experimental intracranial hypertension. J Neurosurg. 1974;41:427-34.

4. Donnelly J, Czosnyka M, Harland S, Varsos GV, Cardim D, Robba C, et al. Cerebral haemodynamics during experimental intracranial hypertension. J Cereb Blood Flow Metab. 2016;37:694-705.

5. Donnelly J, Czosnyka M, Adams H, Cardim D, Kolias AG, Zeiler FA, et al. Twenty-five years of intracranial pressure monitoring after severe traumatic brain injury: a retrospective, single-center analysis. Neurosurgery. 2018. https://doi.org/10.1093/neuros/nyy468.

6. Menon DK. Cerebral protection in severe brain injury: physiological determinants of outcome and their optimisation. Br Med Bull. 1999;55:226-58.

7. Czosnyka M, Whitehouse H, Smielewski P, Kirkpatrick P, Guazzo EP, Pickard JD. Computer supported multimodal bed-side monitoring for neuro intensive care. Int J Clin Monit Comput. 1994;11:223-32.

8. Team RC. R: a language and environment for statistical computing. $R$ Foundation for Statistical Computing, Vienna, Austria. [Internet]. 2015. Available from: http://www.r-project.org/.

9. Wickham H, Francois R. dplyr: A Grammar of data manipulation. [Internet]. R Packag. version 0.5.0. 2016. Available from: http://cran.r-proje ct.org/package $=$ dplyr.

10. Wickham H. Elegant graphics for data analysis. New York: Springer; 2009

11. Wood SN. Fast stable restricted maximum likelihood and marginal likelihood estimation of semiparametric generalized linear models. J R Stat Soc Ser B Stat Methodol. 2011;73:3-36.

12. Ho D, Imai K, King G, Stuart E. Matchlt: Nonparametric Preprocessing for Parametric Causal Inference. J Stat Softw 2011;42:1-28.

13. Lang EW, Kasprowicz M, Smielewski P, Pickard J, Czosnyka M. Changes in cerebral partial oxygen pressure and cerebrovascular reactivity during intracranial pressure plateau waves. Neurocrit Care. 2014;23:85-91.

14. Dias C, Maia I, Cerejo A, Varsos G, Smielewski P, Paiva J-A, et al. Pressures, flow, and brain oxygenation during plateau waves of intracranial pressure. Neurocrit Care. 2014;21:124-32.

15. Liu X, Donnelly J, Czosnyka M, Aries MJH, Brady K, Cardim D, et al. Cerebrovascular pressure reactivity monitoring using wavelet analysis in traumatic brain injury patients: a retrospective study. PLoS Med. 2017;14:e1002348.

16. Donnelly J, Czosnyka M, Adams H, Robba C, Steiner LA, Cardim D, et al. Individualising thresholds of cerebral perfusion pressure using estimated limits of autoregulation. Crit Care Med. 2017;45:1464.
17. Czosnyka M, Aries M, Weersink C, Wolf S, Budohoski K, Dias C, et al. "Solid Red Line": an observational study on death from refractory intracranial hypertension. Acta Neurochir Suppl 2016;122:113-6.

18. Guiza F, Depreitere B, Piper I, Van den Berghe G, Meyfroidt G. Novel methods to predict increased intracranial pressure during intensive care and long-term neurologic outcome after traumatic brain injury: development and validation in a multicenter dataset. Crit Care Med. 2013;41:554-64.

19. Beiner JM, Olgivy CS, DuBois AB. Cerebral blood flow changes in response to elevated intracranial pressure in rabbits and bluefish: a comparative study. Comp Biochem Physiol A Physiol. 1997;1 16:245-52.

20. Rosenthal G, lii JCH, Sorani M, Martin C, Morabito D, Obrist WD, et al. Brain tissue oxygen tension is more indicative of oxygen diffusion than oxygen delivery and metabolism in patients with traumatic brain injury*. Crit Care Med. 2008;36:1917-24.

21. Menon DK, Coles JP, Gupta AK, Fryer TD, Smielewski P, Chatfield DA, et al. Diffusion limited oxygen delivery following head injury. Cri Care Med. 2004;32:1384-90.

22. Ostergaard L, Engedal TS, Aamand R, Mikkelsen R, Iversen NK, Anzabi M, et al. Capillary transit time heterogeneity and flow-metabolism coupling after traumatic brain injury. J Cereb Blood Flow Metab. 2014:34:1585-98.

23. Sekhon MS, Griesdale DE, Czosnyka M, Donnelly J, Liu X, Aries MJ, et al. The effect of red blood cell transfusion on cerebral autoregulation in patients with severe traumatic brain injury. Neurocrit Care. 2015;23:210-6

24. Narotam PK, Morrison JF, Schmidt MD, Nathoo N. Physiological complexity of acute traumatic brain injury in patients treated with a brain oxygen protocol: utility of symbolic regression in predictive modeling of a dynamical system. J Neurotrauma. 2014;31:630-41.

25. Donnelly J, Budohoski KP, Smielewski P, Czosnyka M. Regulation of the cerebral circulation: bedside assessment and clinical implications. Crit Care. 2016;20:129

26. Timofeev I, Czosnyka M, Carpenter KLH, Nortje J, Kirkpatrick PJ, Al-Rawi $P G$, et al. Interaction between brain chemistry and physiology after traumatic brain injury: impact of autoregulation and microdialysis catheter location. J Neurotrauma. 2011;28:849-60.

27. Spiotta AM, Stiefel MF, Gracias VH, Garuffe AM, Kofke WA, Maloney-Wilensky $\mathrm{E}$, et al. Brain tissue oxygen-directed management and outcome in patients with severe traumatic brain injury. J Neurosurg. 2010;113:571-80.

28. Jaeger M, Schuhmann MU, Soehle M, Nagel C, Meixensberger J. Continuous monitoring of cerebrovascular autoregulation after subarachnoid hemorrhage by brain tissue oxygen pressure reactivity and its relation to delayed cerebral infarction. Stroke. 2007;38:981-6.

29. Jaeger M, Dengl M, Meixensberger J, Schuhmann MU. Effects of cerebrovascular pressure reactivity-guided optimization of cerebral perfusion pressure on brain tissue oxygenation after traumatic brain injury. Crit Care Med. 2010;38:1343-7.

30. Jaeger M, Soehle M, Schuhmann MU, Meixensberger J. Clinical significance of impaired cerebrovascular autoregulation after severe aneurysmal subarachnoid hemorrhage. Stroke. 2012;43:2097-101.

31. Löfgren J, von Essen C, Zwetnow NN. The pressure-volume curve of the cerebrospinal fluid space in dogs. Acta Neurol Scand. 1973;49:557-74.

32. Balestreri M, Czosnyka M, Steiner LA, Schmidt E, Smielewski P, Matta $B$, et al. Intracranial hypertension: what additional information can be derived from ICP waveform after head injury? Acta Neurochir (Wien). 2004;146:131-41. 